\title{
Apolipoprotein E Promotes Invasion in Oral Squamous Cell Carcinoma
}

Sangeeta K. Jayakar, ${ }^{*}$ Olivier Loudig, ${ }^{\dagger}$ Margaret Brandwein-Gensler, ${ }^{*}$ Ryung S. Kim, ${ }^{\dagger}$ Thomas J. Ow, ${ }^{* \S}$ Berrin Ustun, ${ }^{*}$ Thomas M. Harris, ${ }^{*}$ Michael B. Prystowsky, ${ }^{*}$ Geoffrey Childs, ${ }^{*}$ Jeffrey E. Segall, ${ }^{*}$ and Thomas J. Belbin ${ }^{*}$

From the Departments of Pathology, ${ }^{*}$ Epidemiology and Population Health, ${ }^{\dagger}$ and Anatomy and Structural Biology, ${ }^{\top}$ Albert Einstein College of Medicine, Bronx, New York; the Department of Pathology and Anatomical Sciences, ${ }^{\ddagger}$ Erie County Medical Center, Buffalo, New York; the Department of Otorhinolaryngology - Head and Neck Surgery, ${ }^{\S}$ Montefiore Medical Center, Bronx, New York; and the Discipline of Oncology," Faculty of Medicine, Memorial University of Newfoundland, St. John's, Newfoundland and Labrador, Canada

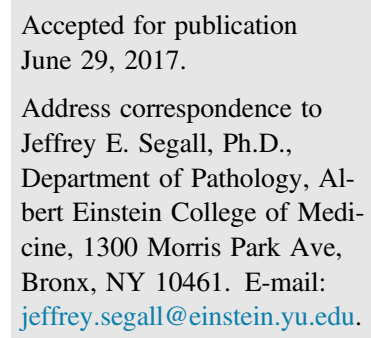

Address correspondence to Jeffrey E. Segall, Ph.D., Department of Pathology, Albert Einstein College of Medicine, 1300 Morris Park Ave, Bronx, NY 10461. E-mail: jeffrey.segall@einstein.yu.edu.

\begin{abstract}
Oral squamous cell carcinoma (OSCC) patients generally have a poor prognosis, because of the invasive nature of these tumors. In comparing transcription profiles between OSCC tumors with a more invasive (worst pattern of tumor invasion 5) versus a less invasive (worst pattern of tumor invasion 3 ) pattern of invasion, we identified a total of 97 genes that were overexpressed at least 1.5 -fold in the more invasive tumor subtype. The most functionally relevant genes were assessed using in vitro invasion assays with an OSCC cell line (UM-SCC-1). Individual siRNA knockdown of 15 of these 45 genes resulted in significant reductions in tumor cell invasion compared to a nontargeting siRNA control. One gene whose knockdown had a strong effect on invasion corresponded to apolipoprotein $\mathrm{E}(A P O E)$. Both matrix degradation and the number of mature invadopodia were significantly decreased with APOE knockdown. $A P O E$ knockdown also resulted in increased cellular cholesterol, consistent with APOE's role in regulating cholesterol efflux. APOE knockdown resulted in decreased levels of phospho-extracellular signalregulated kinase $1 / 2$, phospho-c-Jun $\mathrm{N}$-terminal kinase, and phospho-cJun, as well as decreased activator protein 1 (AP-1) activity. Expression of matrix metalloproteinase 7 (MMP7), an AP-1 target, was also significantly decreased. Our findings suggest that APOE protein plays a significant role in OSCC tumor invasion because of its effects on cellular cholesterol and subsequent effects on cell signaling and AP-1 activity, leading to changes in the expression of invasion-related proteins, including MMP7. (Am J Pathol 2017, 187: 2259-2272; http://dx.doi.org/10.1016/j.ajpath.2017.06.016)
\end{abstract}

Head and neck cancers are the sixth most common malignancy worldwide, with $>650,000$ new cases each year. ${ }^{1}$ Oral cavity squamous cell carcinomas (OSCCs) contribute to 264,000 of these cases, resulting in $>128,000$ deaths annually. ${ }^{1}$ Despite advances in current treatment options, the 5-year survival for head and neck cancer patients has remained at $50 \%$, a number that has not improved in the past 30 years. The poor prognosis associated with OSCC disease is due, in part, to the highly invasive and metastatic abilities of these tumor cells. OSCC patients often present with significant local invasion of tumor through the epithelial basement membrane into the underlying muscle, bone, or salivary tissue. The extent of tumor invasion directly affects patient prognosis and is an important factor in choosing treatment. $^{2,3}$ Depth of invasion of 2 to $4 \mathrm{~mm}$ in the oral tongue or floor of mouth is associated with $15 \%$ to $20 \%$ risk of metastasis to the cervical lymph nodes. ${ }^{4}$ As such, deep invasion into the tongue or mandible often requires extensive resection of oral cavity structures as part of therapy,

\footnotetext{
Supported by the Albert Einstein Cancer Center support grants of the NIH P30CA013330 and CA100324 (J.E.S.), the National Cancer Institute (NCI) grant R21CA104402 (T.J.B.), the NIH/NCI Paul Calabresi Career Development Award for Clinical Oncology 2K12132783-06 (T.J.O.), the NIH/National Center for Advancing Translational Science EinsteinMontefiore Clinical and Translational Science Award grant UL1TR001073 (T.J.O.), and the Departments of Pathology and Epidemiology and Population Health, Albert Einstein College of Medicine and Montefiore Medical Center.

J.E.S. and T.J.B. contributed equally to this work as senior authors.

Disclosures: None declared.
} 
contributing to significant patient morbidity and functional impact on speech and swallowing.

Assessing the extent of tumor invasion using histological parameters in OSCC patients has shown some success in predicting patient outcome. ${ }^{3,5-7}$ Brandwein-Gensler et $\mathrm{al}^{3,5}$ demonstrated that the histological pattern of tumor invasion (POI) contributed positively in predicting OSCC patient outcome as part of their risk assessment model. Specifically, they showed that the worst pattern of tumor invasion (WPOI), identified as the POI score taken from the most invasive portion of the tumor, was significantly associated with overall patient survival as well as local recurrence of disease. $^{3,5}$ This model has since been validated by other groups as a pathological tool for predicting patient outcome, suggesting its potential utility in determining and properly timing treatment options for OSCC patients. ${ }^{6,7}$ Despite the evidence that such clinical and histological parameters might be used to identify tumors that exhibit an invasive phenotype, the underlying molecular events responsible for these characteristics in OSCC are not fully understood.

The search for molecular changes affecting invasion in these tumors has been an active area of research. Herein, we present data to show that tumors that are highly invasive (WPOI5) express higher levels of apolipoprotein E (APOE) protein compared to less invasive tumors (WPOI3), and that this expression of APOE is important to the invasion phenotype of OSCC cells. Mechanistic studies in our model system suggest that APOE decreases the cholesterol content of OSCC cells, leading to changes in intracellular signaling and downstream expression of proteases, and thus contributing to increased tumor cell invasion.

\section{Materials and Methods}

\section{Specimen Collection and Whole-Genome Expression Analysis}

Oral cavity tumor tissue specimens were obtained from Montefiore Medical Center (Bronx, NY). Histological assessment was performed as described, ${ }^{3}$ and samples with WPOI scores of WPOI5 and WPOI3 were identified. Wholegenome gene expression was evaluated in OSCC tumor tissues using two approaches. RNA was extracted from flashfrozen sections of tumors and measured using the HumanHT-12 v4 Beadchip arrays (Illumina, San Diego, CA), as described previously. ${ }^{8,9}$ As an additional evaluation of RNA from the same WPOI5 and WPOI3 tumors, biopsy cores from the edges of the tumors in formalin-fixed, paraffin-embedded tissue blocks were obtained and subjected to RNA extraction. ${ }^{10,11}$ Whole-genome gene expression was then measured using the whole-genome cDNAMediated Annealing, Selection, Extension and Ligation (DASL) array platform (Illumina), as described previously. ${ }^{11}$ Gene expression data were $\log _{2}$ transformed, and a $t$-test was performed comparing the same five WPOI3 and four WPOI5 tumor samples from the both data sets. Genes overexpressed in WPOI5 tumors, as determined by both approaches, were selected that had $P<0.05$, and a minimum fold change of 1.5 in both Beadchip and DASL analyses. The overall falsediscovery rate based on permutation of the group labels was 1\%. All microarray gene expression data were deposited in the National Center for Biotechnology Information Gene Expression Omnibus public data repository (http://www.ncbi. nlm.nih.gov/geo; accession number GSE79795, last accessed October 18, 2016).

\section{Cell Lines and Culture Conditions}

The oral cavity squamous cell carcinoma cell line UM-SCC1 was obtained from Dr. Thomas Carey (University of Michigan, Ann Arbor), and PCI-15B was provided by Dr. Jeffrey Myers (University of Texas MD Anderson Cancer Center, Houston, TX). Cells were grown in Dulbecco's modified Eagle's medium (DMEM)/high-glucose [catalog number (Cat.) SH30243; Hyclone/Thermo Fisher, Waltham, MA] supplemented with $10 \%$ fetal bovine serum (Cat. 16000; Thermo Fisher Scientific), $100 \mathrm{U} / \mathrm{mL}$ penicillin and $100 \mu \mathrm{g} / \mathrm{mL}$ streptomycin (Cat. 15140-122; Thermo Fisher Scientific), $100 \mu \mathrm{mol} / \mathrm{L}$ nonessential amino acids (Cat. 11140-050; Thermo Fisher Scientific), and $2 \mathrm{mmol} / \mathrm{L}$ L-glutamine (Cat. 25030-081; Thermo Fisher Scientific). Cells were grown in medium containing $50 \mu \mathrm{g} / \mathrm{mL}$ Plasmocure (Cat. Ant-pc; InvivoGen, San Diego, CA) for 2 weeks, and then maintained in medium without Plasmocure. All cells were grown at $37^{\circ} \mathrm{C}$ with $5 \% \mathrm{CO}_{2}$.

\section{siRNA Transfection}

Cells were plated at a density of $5 \times 10^{4}$ cells in antibioticfree complete medium for 24 hours before siRNA transfection in 6-well plates in a volume of $2 \mathrm{~mL}$. At the time of transfection, culture medium was replaced with fresh antibiotic-free complete medium containing siGENOME oligos at a final concentration of $50 \mathrm{nmol} / \mathrm{L}$ with $1 \mu \mathrm{L}$ of Dharmafect Reagent 4 (Cat. T-2004-02; GE Dharmacon, Lafayette, CO); cells were incubated for 72 hours without changing medium. Knockdowns were confirmed by Western blot analysis (see below). In the case of JUN knockdowns, cells were incubated at 48 hours before the invasion assay, and knockdowns were confirmed by real-time PCR, as described below. siRNA oligos used were as follows: siGENOME Nontargeting siRNA Pool No. 2, Cat. D-001206-14-05, sequences: 5'-UAAGGCUAUGAAGAGAUAC-3', 5'-AUGUAUUGGCCUGUAUUAG-3', 5'-AUGAACGUGAAUUGCUCAA-3' , and 5'-UGGUUUACAUGUCGACUAA-3'; Human JUN siGENOME SMARTpool, Cat. M-003268-03-0005, sequences: 5'-UGGAAACGACCUUCUAUGA-3', 5'-UAACGCAGCAGUUGCAAAC-3', 5'-GAGCGGACCUUAUGG-

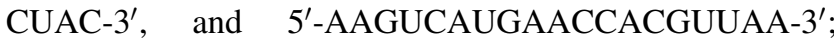
Human matrix metalloproteinase 7 (MMP7) siGENOME SMARTpool, Cat. M-003782-01-0010, sequences: 
5'-GGAACAGGCUCAGGACUAU-3', 5'-GCUCAAGGACUAUCUCAAGA-3'， 5'-GAGAUGCUCACUUCGAUGA$3^{\prime}$, and $5^{\prime}$-CGGAGGAGAUGCUCACUUC-3'; Human APOE siGENOME SMARTpool, Cat. M-006470-00-0005; Human APOE siGENOME siRNA (individual oligos): siAPOE-01, Cat. D-006470-01-0005, sequence: 5'-AGACAGAGCCGGAGCCCGA-3'; siAPOE-02, Cat. D-006470-020005, sequence: 5'-GCGCGGACAUGGAGGACGU-3'; siAPOE-03, Cat. D-006470-03-0010, sequence: 5'GCGCGCGGAUGGAGGAGAU-3'; siAPOE-04, and Cat. D-006470-04-0010, sequence: 5'-CUGCGUUGCUGGUCACAUU-3'. All siRNA oligos were from GE Dharmacon.

\section{In Vitro Invasion Assay}

Invasion assays were performed using $\mathrm{BD}$ BioCoat Matrigel Invasion Chambers (Cat. 08-774-122; BD Biosciences/Fisher, Franklin Lakes, NJ) after siRNA transfection. Invasion chambers were hydrated and equilibrated for 2 hours before addition of cells in DMEM in a 24-well plate, and by adding DMEM inside the chambers with incubation in a $37^{\circ} \mathrm{C}$ incubator. Cells were detached with Accutase (Cat. S-1100-1; BioExpress/Fisher, Kaysville, UT) and counted. OSCC cells were centrifuged, resuspended in serum-free medium $(0.7 \%$ bovine serum albumin/DMEM), and plated into the upper well of the invasion chamber at a density of 100,000 cells in a volume of $0.5 \mathrm{~mL}$. The lower chamber of the transwell assay contained $1 \mathrm{~mL}$ of $0.1 \mathrm{nmol} / \mathrm{L}$ mouse epidermal growth factor (Cat. 53003018; Invitrogen, Carlsbad, CA) diluted in 0.7\% bovine serum albumin/DMEM. Invasion chambers were incubated at $37^{\circ} \mathrm{C}$ for 24 hours. Cells were then fixed with formalin for 15 minutes, and stained with $0.2 \%$ crystal violet for 10 minutes. Cells that did not invade through to the underside of the membrane were removed by scraping. The filters were excised, applied to a glass coverslip, and imaged using a flatbed scanner (Epson America, Long Beach, CA); the percentage area of filter covered by invading cells was quantified using ImageJ software version 1.49 (NIH, Bethesda, MD; http://imagej.nih.gov/ij). For each siRNA experiment, tumor cell invasion was normalized to the percentage invasion of cells treated with a nontargeting siRNA. All individual experiments were performed in triplicate.

\section{RNA Extraction and Real-Time Quantitative RT-PCR from Cultured Cell Lines}

To collect total RNA for expression analysis, cultured cells were washed in ice-cold phosphate-buffered saline, scraped, and lysed in RLT buffer using the RNeasy Mini Kit (Cat. 74104; Qiagen, Valencia, CA). Total RNA was purified, according to the manufacturer's protocol. RNA quality was assessed using an Agilent 2100 BioAnalyzer and an RNA 6000 Nano Kit (Agilent Technologies, Wilmington, DE), according to the manufacturer's instructions. Real-time quantitative RT-PCR was performed using the TaqMan RNA-to-CT 1-Step Kit (Cat. 4392938; Applied Biosystems/
Thermo Fisher Scientific). All gene expression measurements were normalized to the expression of glyceraldehyde3-phosphate dehydrogenase as an internal control.

\section{Preparation of Cell Lysates and Western Blot Analysis}

Cells were washed in cold phosphate-buffered saline, and lysed in a 2X SDS Laemmli buffer containing protease inhibitor (Cat. 11836170-001; Roche, Basel, Switzerland), phosphatase inhibitor cocktail I (Cat. 524624; EMD Chemicals, Billerica, MA), and phosphatase inhibitor cocktail II (Cat. P5726; Sigma, St. Louis, MO). Lysates were passed through a 28-gauge needle to shear DNA, boiled for 5 minutes, and loaded into an SDS-PAGE gel to run for approximately 90 minutes at $120 \mathrm{~V}$. Extracellular APOE protein was measured using $30 \mu \mathrm{L}$ of cell culture supernatant, adding 10X SDS loading buffer, boiling for 5 minutes, and running on gel, as above. Proteins were transferred onto a nitrocellulose membrane (Cat. 1620112; Bio-Rad, Hercules, $\mathrm{CA})$ and then blocked for 1 hour at room temperature in an Odyssey blocking buffer (Cat. 927-40000; LI-COR Biosciences, Lincoln, NE). Primary antibodies were diluted in blocking buffer, according to the manufacturer's instructions, and incubated overnight at $4^{\circ} \mathrm{C}$ on a rocking shaker. Blots were then washed three times in tris-buffered saline with Tween 20, incubated with secondary antibodies IRDye 680RD donkey anti-mouse (1:20,000) (Cat. 926-68072; LI-COR Biosciences) and IRDye 800RD donkey anti-rabbit (1:5000) (Cat. 92632213; LI-COR Biosciences) in blocking buffer for 1 hour at room temperature, washed in tris-buffered saline with Tween 20, and imaged using an Odyssey scanner (LI-COR Biosciences). Images were analyzed using ImageJ software version 1.49. Primary antibodies and their respective dilutions used for Western blot analysis were as follows: apolipoprotein E antibody (1:500) (Cat. Ab52607) from Abcam (Cambridge, UK); P44/42 mitogen-activated protein kinase [MAPK; extracellular signal-regulated kinase (ERK) 1/2] antibody (Cat. 9102S), phospho-p44/42 MAPK (ERK1/2) (Thr202/Tyr204) antibody (Cat. 9101S), c-Jun antibody (Cat. 9165S), phospho-c-Jun (Ser63) antibody (Cat. 2361S), stress-activated protein kinase/ JNK antibody (Cat. 9258S), and phospho-stress-activated protein kinase/JNK (Thr183/Tyr185) antibody (Cat. 4668S), all diluted 1:1000 and from Cell Signaling Technology (Danvers, MA); and $\beta$-tubulin antibody (1:5000) (Cat. T4026) and $\beta$-actin antibody $(1: 20,000)$ (Cat. A5441) from Sigma.

\section{Measurement of Matrix Degradation and Invadopodia}

Matrix degradation and invadopodia assays were performed in glass-bottom MatTek dishes (catalog number P35G-1.5-14-C; MatTek, Ashland, MA) that were coated with Alexa Fluor 405-labeled gelatin (Cat. A-30000; Thermo Fisher Scientific), as described. ${ }^{12,13}$ Cells were plated onto the fluorescently labeled gelatin and allowed to attach for 4 hours. Immunofluorescence was performed, as described previously. ${ }^{13}$ For invadopodia measurements, cells were fixed in $4 \%$ paraformaldehyde for 20 minutes, permeabilized with $0.05 \%$ 
Triton $\mathrm{X}-100$, blocked in $1 \%$ bovine serum albumin/1\% fetal bovine serum, and then incubated with antibodies for cortactin (Cat. 05-180; Millipore) and Tks5 (Cat. Sc-30122; Santa Cruz, Dallas, TX) for 1 hour at room temperature. Cells were incubated with secondary antibodies (Cy5 anti-rabbit and Cy3 anti-mouse) for 1 hour at room temperature. Both cells and matrix were imaged on an inverted Olympus IX71 fluorescent microscope (Olympus America, Center Valley, PA) at 60× objective in the DAPI, tetrarhodamine isothiocyanate, and Cy5 channels to view the Alexa Fluor 405-labeled matrix, cortactin, and Tks5, respectively.

\section{Cholesterol Measurement}

Total cellular cholesterol was measured using the Amplex Red cholesterol assay kit (Cat. A12216; Thermo Fisher Scientific). Cells were lysed in a buffer containing $150 \mathrm{mmol} / \mathrm{L} \mathrm{NaCl}, 1$ $\mathrm{mmol} / \mathrm{L}$ EGTA, $0.1 \mathrm{mmol} / \mathrm{L} \mathrm{MgCl} 2,10 \mathrm{mmol} / \mathrm{L}$ HEPES $(\mathrm{pH}$ 7.4 ), and $0.5 \%$ Triton $X-100$. Protease inhibitor (Roche) was added to the lysis buffer immediately before lysing the cells. Cells were scraped, sonicated for 15 seconds, and then incubated on ice for 30 minutes. Lysates were centrifuged at $4^{\circ} \mathrm{C}$ for 15 minutes at full speed, and supernatant was collected. Cholesterol was quantified in the supernatant, according to the instructions of the manufacturer (Thermo Fisher Scientific). Total protein was quantified in the samples using a Pierce BCA Protein assay (catalog number 23225; Thermo Fisher Scientific) for normalization.

\section{Phospho-Kinase Protein Array}

Changes in the phosphorylation of signaling proteins were screened using a phospho-kinase antibody array (Cat. ARY003B; R\&D Systems, Minneapolis, MN). OSCC cells were transfected with siRNA for APOE, or with a nontargeting siRNA, as described above. After 72 hours, cells were detached with Accutase and counted. Cells were lysed in a volume of $1 \mathrm{~mL}$ lysis buffer per $1 \times 10^{7}$ cells, according to the manufacturer's instructions. Cell lysates were incubated on ice for 30 minutes and then centrifuged at top speed, and protein was quantified using the Pierce BCA Protein assay. Total protein $(600 \mu \mathrm{g})$ from each sample was diluted and incubated with the phospho-kinase array membrane. Membranes were imaged on an Odyssey scanner and analyzed using ImageStudio Lite software version 5.0 (LICOR Biosciences).

\section{Luciferase Assay}

AP-1 transcriptional activity was measured using a luciferase reporter construct provided by Dr. Ahmad Waseem (Queen Mary University of London, London, England). OSCC cells were initially transfected with siRNA for APOE-04 or nontargeting siRNA control. Forty-eight hours after initial siRNA transfection, cells were then transfected with $1.98 \mu \mathrm{g}$ of a firefly luciferase reporter construct containing six repeats of a consensus AP-1 binding motif in the pGL4.26 vector (Promega, Madison, WI), as described in Brown et al, ${ }^{14}$ along with $0.02 \mu \mathrm{g}$ of the Renilla luciferase construct pRL-CMV (Promega). FuGENE HD (Cat. PRE2311; Promega) was used as a transfection reagent. After 24 hours, the cells were lysed and luciferase signal was measured using a dualluciferase reporter assay, according to the manufacturer's instructions (Cat. PR-E1910; Promega).

\section{RNA Extraction and Real-Time Quantitative RT-PCR from Cultured Cell Lines}

To collect total RNA for expression analysis, cultured cells were washed in ice-cold phosphate-buffered saline, scraped, and lysed in RLT lysis buffer using the RNeasy Mini Kit (Cat. 74104; Qiagen). Total RNA was purified according to the manufacturer's protocol. RNA quality was assessed using an Agilent 2100 BioAnalyzer and RNA 6000 Nano Kit (Agilent Technologies, Wilmington, DE), according to the manufacturer's instructions. Real-time quantitative RTPCR was performed using the TaqMan RNA-to-CT 1-Step Kit. All gene expression measurements were normalized to the expression of glyceraldehyde-3-phosphate dehydrogenase as an internal control.

\section{Library Preparation and RNA-Seq Analysis}

After validating reduced expression of APOE mRNA and assessing RNA quality for each sample, 20 to $50 \mu \mathrm{g}$ of total RNA was provided to the New York Genome Center for RNA-sequencing (RNA-Seq) analysis. Two independent RNA preparations were used for the UMSCC-1 cell line, each containing a sample treated with siNT and siAPOE, for a total of four samples. RNA sequencing libraries were prepared using the Illumina TruSeq Stranded mRNA Sample Preparation Kit. Briefly, $500 \mathrm{ng}$ of total RNA was purified by oligo-dT beads selecting for polyadenylated RNA species and fragmented by divalent cations under elevated temperature. The fragmented RNA underwent first-strand synthesis using reverse transcriptase with random priming. Second-strand synthesis was performed using DNA polymerase I and RNaseH. All cDNA fragments were subjected to end repair, adenylation of the $3^{\prime}$ ends, and ligation of adapters. The resulting cDNA libraries were enriched using eight cycles of PCR. Quality control of the library construction process consisted of assaying the final library size using the Agilent Bioanalyzer and quantifying the final library content by real-time PCR and PicoGreen (fluorescence) methods. A single observed peak between 250 and $350 \mathrm{bp}$ indicated a properly constructed and amplified library ready for sequencing.

RNA sequencing was performed on the HiSeq 2500 instrument using v4 SBS chemistry, according to the Illumina protocol, as described. ${ }^{15}$ Sequencing libraries were loaded onto the HiSeq 2500 flow cell for clustering on the cBot using the instrument-specific clustering protocol. Sequencing reads were aligned with $\mathrm{STAR}^{16}$ version 2.4.0c, 

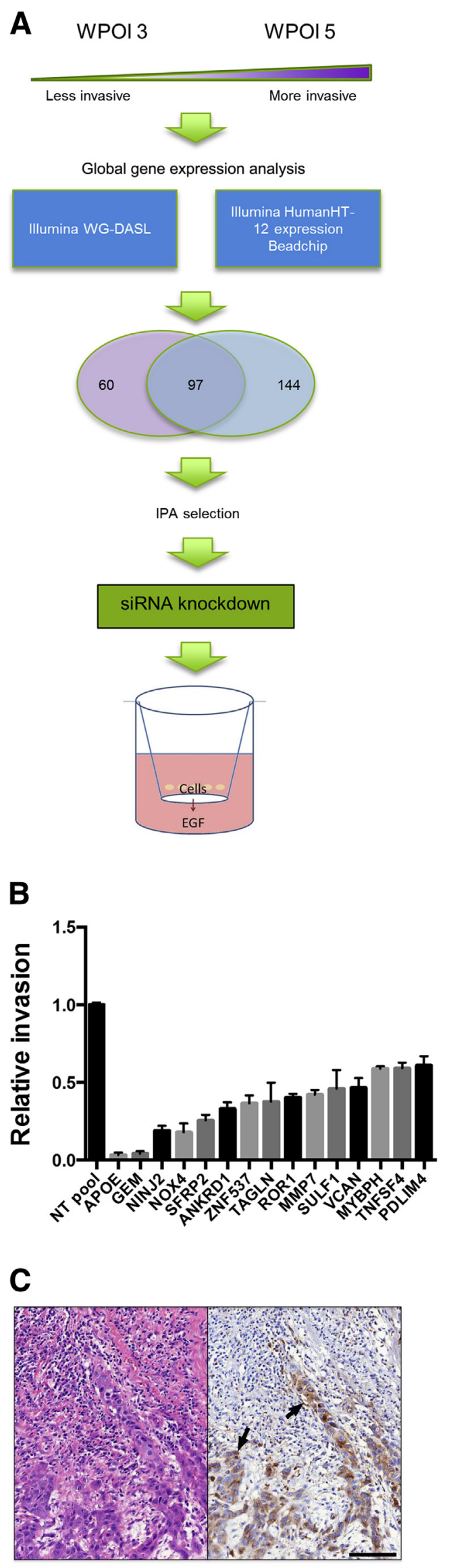

and genes annotated in Gencode v18 were quantified with featureCounts ${ }^{17}$ v1.4.3-p1. The DESeq2 package in Bioconductor was used for normalization. ${ }^{18}$

\section{Analysis of Differentially Expressed Genes and IPA}

Identification of differentially expressed genes in response to $A P O E$ knockdown used the log-transformed RNA-Seq data from DESeq2, as described above. For each of the two experiments, we calculated the difference in expression of siNT minus SIAPOE cells for all genes; these values were referred to as the pairwise $\Delta$ values. We then averaged the two $\Delta$ values and assessed significance of the expression change using a z-test. All data were then sorted on the basis of the magnitude of the pairwise $\Delta$ values, such that genes with the highest values of pairwise $\Delta$ values identified as the most down-regulated with knockdown of APOE, and those with the lowest $\Delta$ values were genes most up-regulated with $A P O E$ knockdown. For the other analyses performed in Ingenuity Pathway Analysis (IPA; build version 400896M, content version 27821452; Qiagen, Germantown, MD), we uploaded expression data for the average $\Delta$ values and their corresponding z-tests for the 57,445 IDs from RNA-Seq analysis. A total of 32,536 IDs corresponded with mapped IDs in IPA. Genes defined as differentially expressed were those with a log expression ratio of at least 0.75 and a cutoff for the expression $P<0.005$ and $\log _{2}$ expression greater than three. This yielded 548 targets, which were used for all of the subsequent IPA analyses. For gene set enrichment analysis, genes with $\log _{2}$ expression values greater than four were ranked according to the average $\log _{2}$ change in expression and analyzed using the gene set enrichment Preranked option with the c3.tft.v5.1.symbols.gmt signature and weighted enrichment for 1000 permutations.

\section{Cholesterol Treatment}

OSCC cells were incubated for 48 hours before cholesterol treatment, and grown to $70 \%$ confluency. Water-soluble cholesterol (Cat. C4951; Sigma) was dissolved in DMEM

\footnotetext{
Figure 1 Screening strategy for invasion-related genes. A: Wholegenome expression data sets from the same worst pattern of tumor invasion (WPOI) 3 (low invasion) and WPOI5 (high invasion) oral squamous cell carcinoma (OSCC) tumors were compared by two approaches in parallel: using biopsy cores with the Illumina WG-DASL platform and using flash-frozen tumor tissue with the Illumina HT-12 Beadchip platform. A total of 97 genes overexpressed in WPOI5 tumors in both comparisons were assessed by Ingenuity Pathway Analysis (IPA) software version 9.0 (content version 3210 ), and 45 genes were selected for testing with an in vitro invasion assay. B: Genes with the greatest effect on tumor cell invasion on knockdown were measured using the in vitro invasion assay. All shown knockdowns were considered significant compared to the nontargeting control cells, as determined by analysis of variance. C: Apolipoprotein E (APOE) immunohistochemical staining of an OSCC primary tumor sample shows high APOE staining in tumor cells at the invading edge, as indicated by arrows. Data are expressed as means + SEM for triplicate measurements (B). Scale bar $=100 \mu \mathrm{m}$. EGF, epidermal growth factor; NT, nontargeting.
} 
containing $10 \%$ fetal bovine serum to a final concentration of $0.8 \mathrm{mg} / \mathrm{mL}$, filter sterilized, and preheated to $37^{\circ} \mathrm{C}$. Culture medium was aspirated and replaced with cholesterol-containing medium for 60 minutes. For the control group, medium was aspirated and replaced with fresh complete medium, omitting cholesterol for the same time period. After incubation, cells were then placed on ice and processed for Western blot analysis, as described above.

\section{Analysis of RNA-Seq Data from The Cancer Genome Atlas}

Analysis of RNA-Seq data from The Cancer Genome Atlas used 69 human papilloma virus-negative tumor samples, labeled as oral cavity, that were included in the January 2015 report describing the comprehensive genomic characterization of head and neck squamous cell carcinomas. ${ }^{19}$ RNA-Seq counts for APOE and MMP7 were log transformed and used to compute correlation between APOE and MMP7 in SPSS version 22 (IBM, Armonk, NY).

\section{Results}

Whole-Genome Expression Analysis Identifies Novel Overexpressed Genes in Highly Invasive WPOI5 OSCC Tumors

Invasion is a key event in oral squamous cell carcinoma tumors that contributes to poor patient survival. We set out to identify genes that were correlated with the invasive phenotype by examining genes that were overexpressed in highly invasive tumors compared to less invasive tumors (Figure 1A). We measured global gene expression from nine OSCC tumors that had been examined by a pathologist (M.B.-G.) on surgical removal and given a score for the WPOI criterion. Four of these tumors were characterized as WPOI5 (highly invasive), and five tumors were characterized as WPOI3 (less invasive). We examined whole-genome expression data for each tumor using two independent approaches, one obtained using the Illumina HT-12 platform with RNA obtained from flash-frozen primary tumor tissue, and one obtained using the Illumina DASL protocol using RNA from cores taken from the tumor edge of formalinfixed, paraffin-embedded tissues. From these parallel data sets, 111 Beadchip probes corresponding to 97 genes were identified by both approaches as overexpressed at least 1.5fold in WPOI5 tumors compared to WPOI3 tumors, with a $P<0.05$ and a false-discovery rate of $<1 \%$.

Overexpression of these genes in the more invasive WPOI5 tumors suggests that they may play a role in driving the invasion process. When surveyed for functional relevance by IPA version 9.0 (content version 3210), we found that 45 of the 97 identified genes clustered into at least one of the top five functional categories that included cellular assembly, cellular movement, cell morphology, cell death, and cell-to-cell signaling (Supplemental Table S1). We, therefore, decided to focus on this subset of 45 genes for downstream functional analysis.

\section{Systematic siRNA Knockdown Identifies a Subset of Overexpressed Genes That Significantly Affect OSCC Tumor Cell Invasion}

Each of the 45 genes identified above was individually tested for its effect on tumor cell invasion using an in vitro transwell invasion assay with the human OSCC cell line UM-SCC-1. Each gene was transiently knocked down for 72 hours using a siGENOME SmartPool (GE Dharmacon) containing four different siRNA sequences. A nontargeting siRNA pool was used as a negative control. From this screen, a subset of 15 genes was identified whose knockdown significantly decreased the invasion of the UM-SCC-1 cells (Figure 1B). Surprisingly, the gene whose knockdown had the most significant effect on tumor cell invasion coded for APOE. Moreover, immunohistochemical staining of OSCC tumors, using an antibody for APOE protein, showed high APOE staining at the invading edges of tumors, a pattern consistent with a role for this protein in tumor cell invasion (Figure 1C). To eliminate the possibility of an siRNA off-target effect, we obtained the four individual siRNA sequences that made up the original siRNA SmartPool used above. All four individual siRNA sequences resulted in knockdown of protein (Figure 2A), as well as a significant reduction in tumor cell invasion compared to the nontargeting control (Figure 2, B and C). Furthermore, we selected two of the four siRNAs (siAPOE-3 and siAPOE-4) and confirmed in a second OSCC cell line (PCI15B) that knockdown of APOE resulted in significant decrease in tumor cell invasion compared to the nontargeting control (Figure 2D). In all cases, we observed no significant decrease in tumor cell proliferation in response to APOE knockdown (data not shown). Having demonstrated the consistency of the result, all subsequent assays were performed in UM-SCC-1 cells with knockdown of APOE using a single siRNA (siAPOE-4).

\section{Knockdown of $A P O E$ Results in Decreases in Matrix Degradation and Mature Invadopodia}

One mechanism by which $A P O E$ knockdown might be affecting tumor cell invasion is through the inhibition of matrix degradation. We tested this hypothesis in two ways: first, by measuring degradation of fluorescently labeled gelatin matrix, and second, by staining for invadopodium protein markers, as invadopodia represent the major architectural effectors of matrix degradation (Figure 3). Using UM-SCC-1 cells treated with siRNA targeting APOE or a nontargeting siRNA, we evaluated average matrix degradation area per cell. We observed that cells with decreased levels of APOE protein had significantly lower levels of matrix degradation compared to nontargeting control cells (Figure 3, A and B). Second, in assessing the levels of 
invadopodium protein markers, we quantified the number of mature invadopodia as the number of degradation holes in the gelatin matrix that colocalized with the invadopodium markers cortactin and Tks5. Areas of colocalization of cortactin and Tks5 without degradation holes were counted as invadopodium precursors. Knockdown of APOE resulted in a significant decrease in the number of mature invadopodia compared to the nontargeting control cells (Figure 3, $\mathrm{A}$ and $\mathrm{C}$ ). However, the number of invadopodium precursors was not significantly altered by APOE knockdown. The reduced numbers of mature invadopodia with concomitant reduction in matrix degradation are consistent with the reduced invasion seen with $A P O E$ knockdown.

\section{APOE Knockdown Results in Decreased Phosphorylation of ERK, cJun, and JNK, and Decreased AP-1 Activity}

We hypothesized that alterations in tumor cell signaling associated with APOE protein levels likely play an important role in the observed OSCC invasion phenotype. To test this hypothesis, we used a phospho-kinase antibody array that screened 34 individual phosphorylated protein candidates to measure alterations in phospho-protein levels in siAPOE-4-treated UM-SCC-1 cells compared to the nontargeting siRNA-treated control cells. In replicate antibody array experiments, the largest relative changes we observed in both experiments were decreased levels of phospho-cJun (Ser63), phospho-ERK1/2 (T202/Y204, T185/Y187), phospho-STAT3 (S727), and phospho-p38 $\alpha$ (T180/Y182) in the siAPOE-4-treated cells compared to the nontargeting siRNA-treated control cells (Supplemental Figure S1). We confirmed the decrease in phosphorylation of ERK1/2 and cJun by Western blot analyses using global protein extracts obtained from these UM-SCC-1 cells. Knockdown of APOE was associated with significant reduction in the levels of phospho-ERK1/2 relative to total ERK1/2 protein (Figure 4A), as well as significant reduction in the levels of phospho-cJun (Ser63) relative to total cJun (Figure 4B). Although phospho-JNK was tested on the phospho-kinase protein array, its signal was extremely low. As it is known that JNK can phosphorylate cJun, we decided to test the levels of phospho-JNK by Western blot analysis. Indeed, we also observed a significant decrease in phospho-JNK in UM-SCC-1 cells in response to APOE knockdown (Figure 4C). As we could not confirm the differences in phospho-STAT3 or phospho-p38 $\alpha$ by Western blot analysis, these proteins were eliminated from subsequent experiments.

As cJun is a subunit of AP-1, a transcription factor complex deeply implicated in regulating the invasive response to tumor cells, we hypothesized that the decrease in phospho-cJun in response to APOE knockdown might also translate to a decrease in AP-1 activity. To test AP-1 activity in OSCC cells, we used a reporter construct containing six repeats of the AP-1 binding sequence upstream of a firefly luciferase reporter gene, and cotransfected a Renilla luciferase construct as a normalization control. We observed that AP-1 activity was decreased in UM-SCC-1 cells in response to APOE knockdown (Figure 4D). Knockdown of $c J u n$ expression with siJUN yielded a $50 \%$ knockdown of cJUN when measured by TaqMan real-time PCR (Supplemental Figure S2). Furthermore, siRNA knockdown of cJun significantly impaired tumor cell invasion in UM-SCC-1 compared to the nontargeting control cells (Figure 4E), consistent with a mechanism in which loss of activated $c J u n$ reduces AP-1 transcription factor activity with a corresponding effect on tumor invasion.
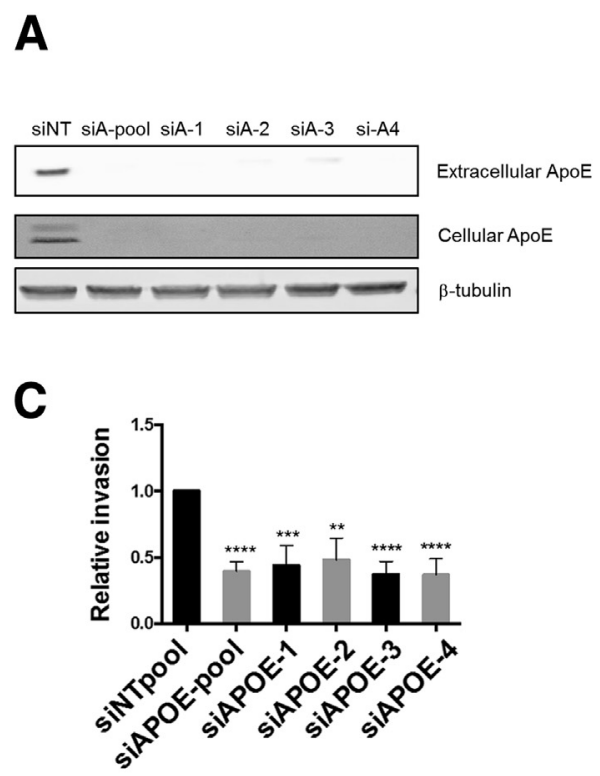

B

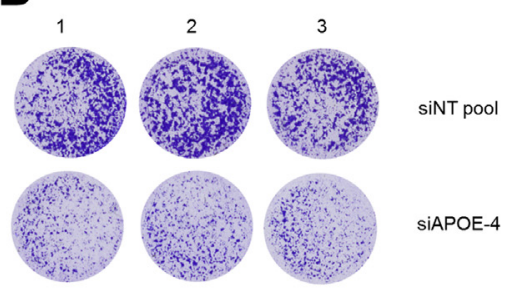

D

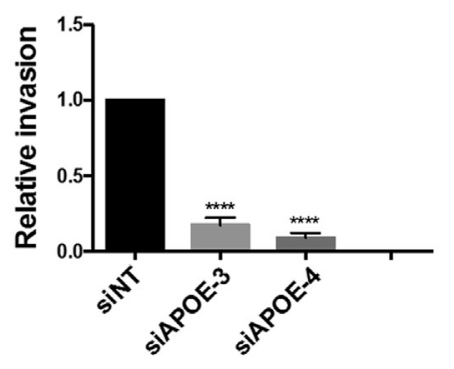

Figure 2 A: Knockdown of apolipoprotein $E$ $(A P O E)$ results in decreased in vitro tumor cell invasion of UM-SCC-1 and PCI-15B. Representative Western blot analyses of APOE protein expression in UM-SCC-1 after APOE knockdown by siRNA oligos. APOE protein levels in both tumor cell lysates (cellular) and secreted APOE in the supernatant (extracellular) were assayed. $\beta$-Tubulin was used as a loading control for cellular APOE. B: Representative images of in vitro invasion chamber filters after treatment of UM-SCC-1 cells with either SIAPOE-4 or the SINT nontargeting control. C: Quantitation of invasion of UM-SCC-1 cells after treatment with nontargeting siRNA, pooled APOE siRNA (siA-pool), or individual APOE siRNAs (siA-1 to siA-4). D: Reduced in vitro tumor cell invasion of PCI15B cells in response to $0.1 \mathrm{nmol} / \mathrm{L}$ epidermal growth factor treated with SiRNA SiAPOE-3 or SiAPOE-4 compared to nontargeting siRNA (siNT pool). Percentage knockdown of APOE mRNA as measured by real-time PCR was $83 \%$ and $90 \%$ for SIAPOE-3 and SIAPOE-4, respectively. Data are expressed as means + SEM for triplicate measurements (C). ${ }^{* *} P<0.01,{ }^{* *} P<0.001$, and $* * * * P<0.0001$ (z-test) versus the siNT sample. 
A

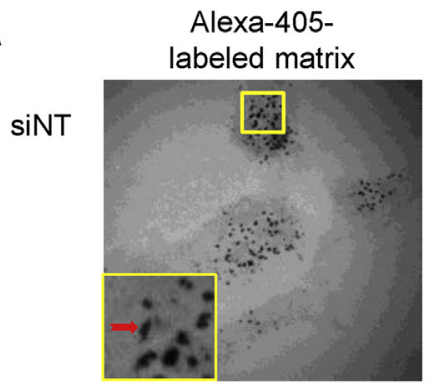

SIAPOE

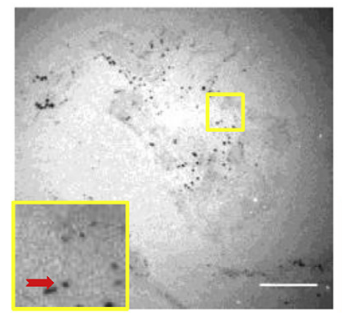

B

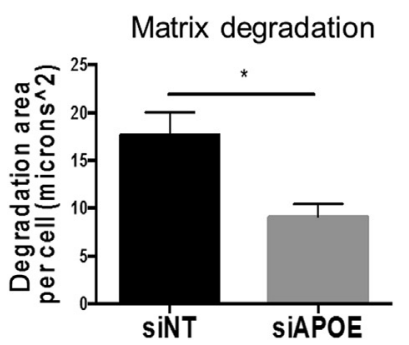

Cortactin
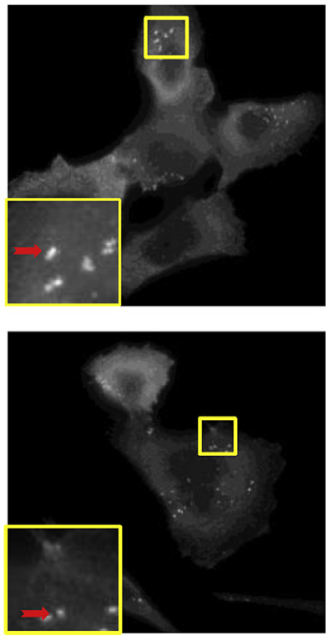

C
Number of invadopodia

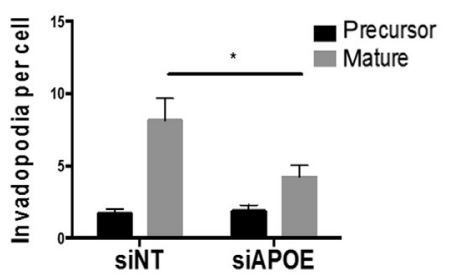

Figure 3 Apolipoprotein E (APOE) knockdown reduces matrix degradation and number of mature invadopodia. A: Representative images of matrix degradation and invadopodium markers cortactin and Tks5 in UM-SCC-1 cells. Boxed areas are shown at higher magnification in the insets. Mature invadopodia, defined by a colocalization of cortactin and Tks5 with a degradation hole in the matrix, are indicated by arrows in the insets. B: Matrix degradation area per cell in SiAPOE-treated versus nontargeting siRNA control for UMSCC-1 cells. C: Quantification of invadopodia in SiAPOEtreated versus nontargeting siRNA control for UM-SCC-1 cells. Counts of mature invadopodia used the above definition, whereas invadopodium precursors were defined as colocalization of cortactin and Tks5 without a degradation hole in the gelatin matrix. Ten random fields were imaged and used for analysis in each of triplicate experiments. Data are expressed as means + SEM for triplicate measurements (B and C). ${ }^{*} P<0.05$ ( $t$-test). Scale bar $=20 \mu \mathrm{m}$. Original magnification, $\times 3.7$ (insets).
MMP7 Plays a Role in Invasion of OSCC Cells, and Has Reduced mRNA Levels with APOE Knockdown

The AP-1 transcription factor is known to regulate the expression of genes associated with a wide variety of biological functions, including tumor cell invasion. ${ }^{20}$ It has also been implicated in metastasis of head and neck squamous cell cancer tumors. ${ }^{21}$ Interestingly, $M M P 7$ was 1 of the original 15 genes identified in our whole-genome screen for invasion-related genes (Supplemental Table S1 and Figure 1B), and has been shown to be directly regulated by AP-1. ${ }^{22-25}$ Moreover, MMP7 protein has been shown to play an important role in the invasion and metastasis of other cancers by increasing matrix degradation. ${ }^{26,27} \mathrm{We}$, therefore, tested the hypothesis that $M M P 7$ expression could be affected by the levels of APOE protein, via a mechanism whereby AP-1 contributes to its transcriptional regulation. In UM-SCC-1 cells, we observed that siRNA knockdown of $A P O E$ was accompanied by a significant decrease in MMP7 mRNA (Figure 5A). In addition, MMP7 mRNA expression was significantly decreased with siRNA knockdown of cJun (Figure 5B), thereby supporting the hypothesis that cJun, and thus AP-1, can regulate expression of $M M P 7$. Finally, direct siRNA knockdown of $M M P 7$ significantly impaired tumor cell invasion in UM-SCC-1 cells (Figure 5C).
To investigate the clinical relevance of $A P O E$ expression and downstream $M M P 7$ expression with human pathological specimens, we examined mRNA expression of both genes in primary tumors from two cohorts of oral cavity cancer patients: Beadchip expression data from 37 human papilloma virus-negative oral cavity cancer patients undergoing treatment at Einstein-Montefiore Medical Center (Bronx, NY), and RNA-Seq expression data from 69 human papilloma virus-negative oral cavity cancer patients obtained from The Cancer Genome Atlas. We found a significant positive correlation between APOE mRNA expression (ILMN_1740938) and MMP7 mRNA expression (both ILMN_1685403 and ILMN_2192072 probes) in the Einstein-Montefiore OSCC patient cohort [Pearson coefficient values of $0.67(P<0.0001)$ and $0.71(P<0.0001)$, respectively]. In addition, a significant positive correlation was observed between APOE mRNA expression (APOE; gene ID, 348) and MMP7 mRNA expression (MMP7; gene ID, 4316) in normalized RNA-Seq data from The Cancer Genome Atlas oral cancer cohort (Pearson coefficient value $=0.5185, P<0.0001)$ (Figure $5 \mathrm{D})$. Together, these results confirm that our screening approach and findings in an initial small set of OSCC tumors could be globally correlated in two larger patient cohorts, and that APOE protein may play an important role in tumor cell invasion of OSCC cells. 
A

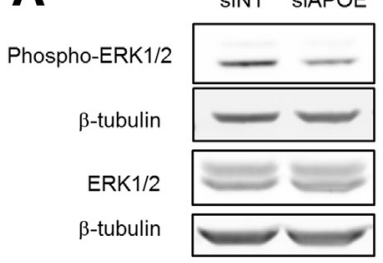

C

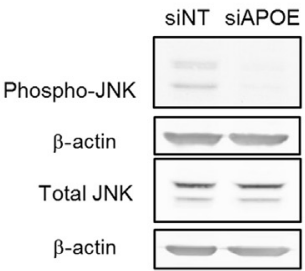

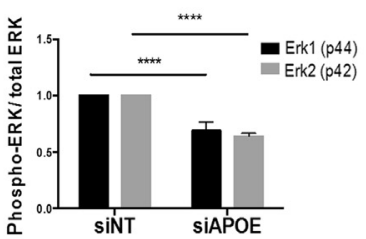

.

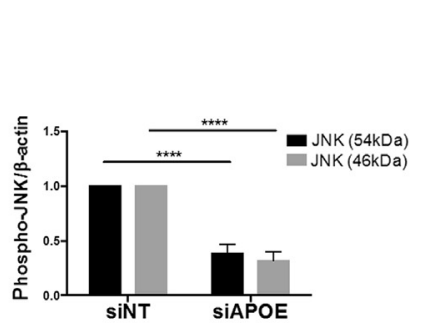

B
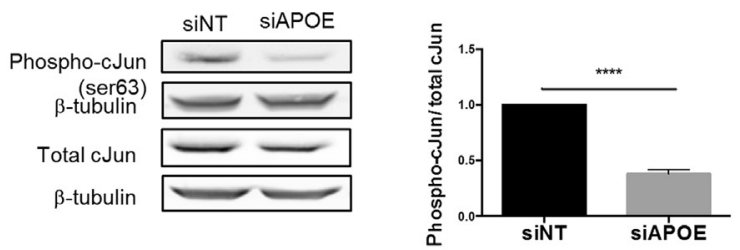

E

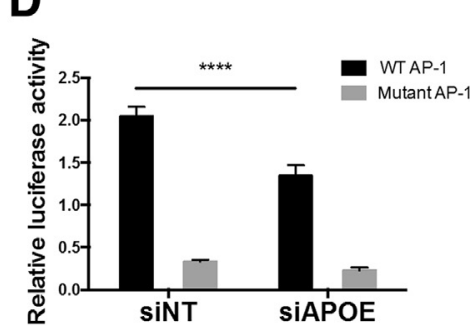

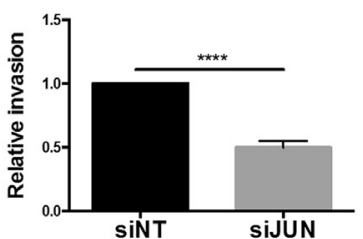

Figure 4 Knockdown of apolipoprotein E (APOE) decreases phosphorylation of extracellular signal-regulated kinase (ERK), cJun, and JNK, and decreases AP-1 activity. A: Representative Western blot analysis and quantitation of phosphorylated ERK and total ERK after treatment with either siNT or siAPOE in UMSCC-1 cells. Quantitation is shown as signal of phosphorylated ERK relative to total ERK, normalized to $\beta$-tubulin. B: Representative Western blot analysis and quantitation of phosphorylated JNK and total JNK after treatment with siNT or siAPOE in UM-SCC-1 cells. Quantitation is shown as signal of phosphorylated JNK relative to $\beta$-actin. A z-test was used. C: Representative Western blot and quantitation of phosphorylated cJun (Ser63) and total cJun after treatment with either siNT or SIAPOE in UM-SCC-1 cells. Quantitation is shown as signal of phosphorylated cJun relative to total cJun, normalized to $\beta$-tubulin. D: Quantitation of AP-1 activity in UM-SCC-1 cells using a luciferase reporter construct with a minimal promoter and six repeats of AP-1 binding domain [wild-type (WT) AP1] or a mutated AP-1 binding domain (mutant AP1). Signals were normalized to the signal obtained from a cotransfected Renilla luciferase reporter construct. A $t$ test was used. E: In vitro invasion of UM-SCC-1 cells in response to $0.1 \mathrm{nmol} / \mathrm{L}$ epidermal growth factor after siRNA knockdown of JUN expression. A z-test was used. Data are expressed as means + SEM for triplicate measurements. $n=3(\mathbf{A}-\mathbf{E}) .{ }^{* * * *} P<0.0001$.

\section{Whole Transcriptome Analysis Identifies Differentially Expressed Upstream Regulators and Signaling Pathways That Can Mediate the Effect on Tumor Cell Invasion Resulting from APOE Knockdown}

In collaboration with the New York Genome Center, we used a whole transcriptome analysis with RNA-Seq technology to identify differentially expressed genes that could be grouped into canonical pathways using IPA. Knockdown of APOE expression with siAPOE-4 yielded $>90 \%$ knockdown of APOE when measured by TaqMan real-time PCR (Supplemental Table S2). From these replicate experiments, 548 differentially expressed genes $\left(\log _{2}\right.$ change of at least $0.75, P \leq 0.005)$ were identified. Interestingly, genes showing significantly decreased expression in response to APOE knockdown included receptor tyrosine kinase AXL, dickkopf WNT signaling pathway inhibitor 3 , and transgelin, all of which were among the 45 initially identified genes overexpressed in the WPOI5 tumors; their expression has been previously associated with invasion, metastasis, and poor prognosis in head and neck squamous cell cancer. ${ }^{28-30}$ IPA of the gene sets to assess upstream regulators that might be affected by $A P O E$ knockdown revealed transcription factors, signal transduction molecules, and growth factors whose deactivation with $A P O E$ knockdown was supported by clusters of gene expression changes observed in RNA-Seq data (Supplemental Table S3). These analyses identified ERK, Jnk, and Ap1 as upstream regulators associated with a high APOE phenotype, a finding that validated our data that AP-1 activity was reduced with APOE knockdown (Figure 6 and Supplemental Table S3). Other significant gene clusters included those corresponding to TGFB1, HGF, NF- $\kappa \mathrm{B}$, and Vegf. Gene set enrichment analysis confirmed the effect on AP-1 activity of APOE knockdown: from the transcription factor target gene sets, AP1 motif-containing genes were the most significantly altered (false-discovery rate, <0.001) (Supplemental Figure S3).

The genes that were differentially expressed with APOE knockdown were also grouped into canonical pathways using IPA. Selected top canonical pathways that were significantly affected are displayed in Supplemental Table S4. As expected, the ERK/MAPK signaling pathway was significantly affected. Other interesting canonical pathways that were highlighted included integrin signaling, NF- $\mathrm{BB}$ signaling, phosphatidylinositol 3-kinase/AKT signaling, and several cytokine signaling pathways, including IL-8 signaling, IL-6 signaling, and C-X-C chemokine receptor 4 signaling. Differentially expressed genes were also clustered into common disease or functional categories using IPA (Supplemental Table S5). Strikingly, the top four functional categories, as ranked by activation z-score, were related to tumor cell invasion or migration.

In summary, the whole transcriptome analysis confirms altered AP-1 signaling as a major effect of APOE knockdown, and cell migration and invasion as major functional effects of $A P O E$ knockdown. 

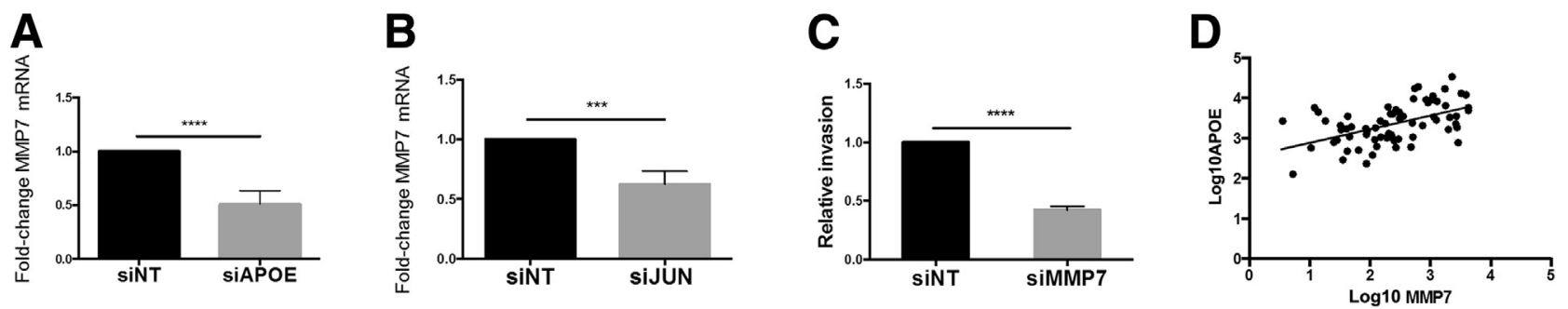

Figure 5 Matrix metalloproteinase 7 (MMP7) expression is regulated by apolipoprotein E (APOE) and contributes to tumor cell invasion. A: Relative fold change in MMP7 mRNA expression in UM-SCC-1 cells after siRNA knockdown of APOE. B: Relative fold change in MMP7 mRNA expression in UM-SCC-1 cells after knockdown of cJun. C: In vitro invasion of UM-SCC-1 cells in response to $0.1 \mathrm{nmol} / \mathrm{L}$ epidermal growth factor after siRNA treatment to reduce expression of MMP7. D: Scatter plot of APOE expression and MMP7 expression in oral squamous cell carcinoma tumor RNA-sequencing data from The Cancer Genome Atlas database. $r=0.519, P<0.0001$. Data are expressed as means + SEM for triplicate measurements (A-C). $n=69(\mathbf{D}) .{ }^{* * * P} P 0.001,{ }^{* * * * P}<0.0001$ (z-test).

\section{APOE Knockdown Results in Increased Total Cellular Cholesterol, and Contributes to Decreased Phosphorylation of ERK}

APOE protein is known to play a role in both the regulation of cellular cholesterol levels and cellular cholesterol efflux. ${ }^{31,32}$ Cellular cholesterol has also been shown to have profound effects on cellular signaling, including on levels of phosphorylated ERK. ${ }^{33-36}$ To investigate a possible role for cellular cholesterol in OSCC invasion, we measured total cellular cholesterol in response to $A P O E$ knockdown. We found that UM-SCC-1 cells showed a significant increase in total cellular cholesterol in response to $A P O E$ knockdown (Figure 7A). In addition, treatment of UM-SCC-1 cells with cholesterol resulted in a significant decrease in phosphoERK (Figure 7, B and C). Taken together, these results suggest a novel mechanism whereby the more invasive tumor phenotype associated with higher levels of APOE protein may be because of increased cholesterol efflux, resulting in enhanced ERK and JNK signaling and
AP-1-induced transcription of $M M P 7$ and other genes involved in tumor cell invasion.

\section{Discussion}

A comparison of whole genome transcriptional profiles of OSCC tumors on the basis of histopathological features and the WPOI score was successful in identifying genes that play a key role in tumor cell invasion. We identified $A P O E$ in an initial screen for genes whose expression correlated with pattern of invasion in OSCC tumors, and were able to demonstrate its importance to tumor cell invasion in a human OSCC cell line. Knockdown of APOE expression decreased the ability of UM-SCC-1 cells to degrade extracellular matrix, and decreased the number of mature invadopodia without significantly affecting invadopodium precursors. In addition, knockdown of $A P O E$ decreased the amount of phosphorylated ERK, cJUN, and JNK, leading to a decrease in AP-1 activity. AP-1 downstream target $M M P 7$ expression was identified in our initial screen of genes associated with WPOI,

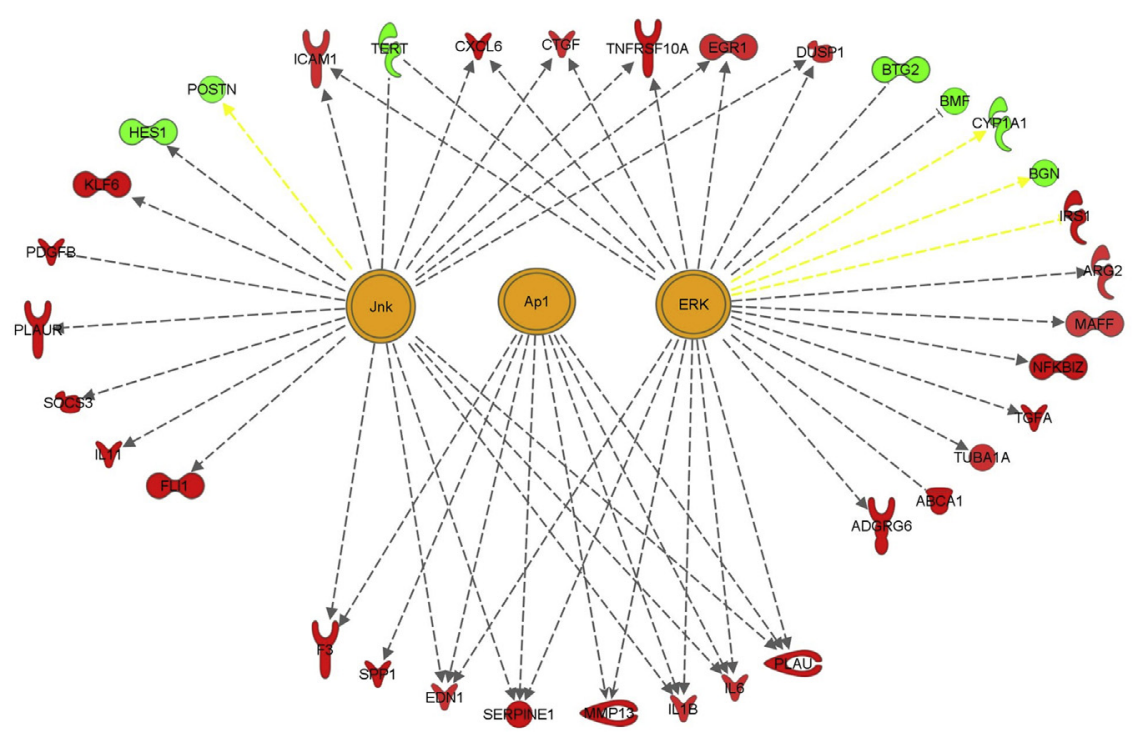

Figure 6 Upstream regulator networks for $E R K$, $J N K$, and $A P 1$, as mapped by Ingenuity Pathway Analysis. Arrowheads indicate positive activation, whereas bars indicate suppression. Red gene symbols indicate the genes that were downregulated with $A P O E$ knockdown, whereas green indicates up-regulation. Line endings indicate published literature interactions (arrows for positive, blocked for negative, open for not specified). Gray lines indicate gene changes consistent with published interactions, whereas yellow lines indicate gene changes inconsistent with published interactions. 

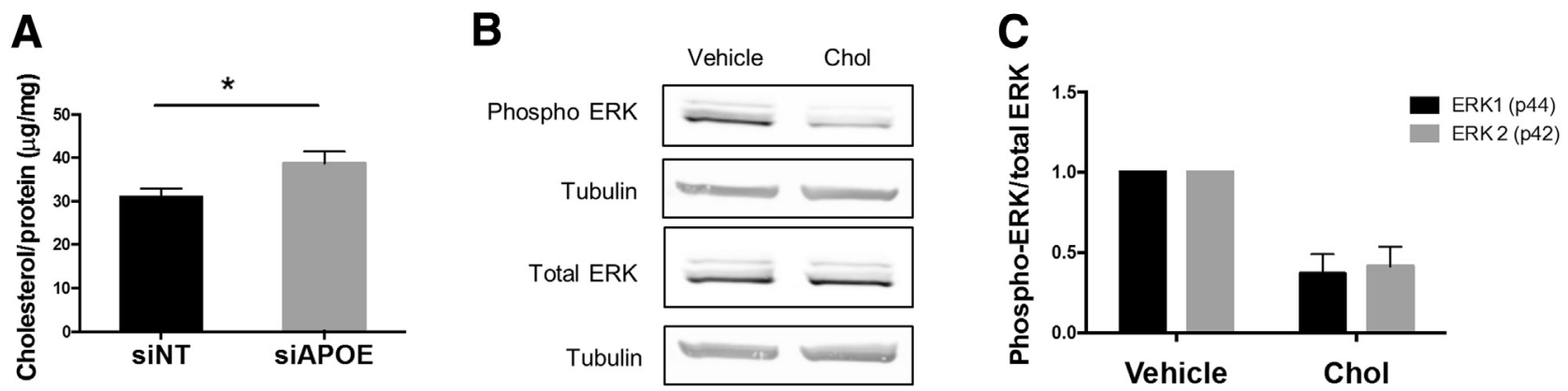

Figure 7 Cholesterol treatment of oral squamous cell carcinoma cells decreases phosphorylated extracellular signal-regulated kinase (ERK). A: Measurements of total cellular cholesterol after knockdown of APOE in UM-SCC-1 cells. B: Western blot analysis of phosphorylated ERK and total ERK after cholesterol (Chol) treatment of UM-SCC-1 cells (Chol, $0.8 \mathrm{mg} / \mathrm{mL}$, versus vehicle control). C: Quantitation of phospho-ERK relative to total ERK, normalized to $\beta$-tubulin, in UM-SCC-1 cells. Relative phospho-ERK levels in the cholesterol-treated cells have been normalized to the vehicle-treated control cells for each experiment. Data are expressed as means + SEMs for triplicate measurements $(\mathbf{A}$ and $\mathbf{C})$. ${ }^{*} P<0.05$ (z-test).

and we demonstrated that MMP7 mRNA levels were decreased in response to APOE knockdown. APOE knockdown also resulted in increased cellular cholesterol, and adding exogenous cholesterol to UM-SCC-1 cells led to decreased ERK phosphorylation. In summary, our data support a model whereby oral cavity cancer tumors with higher expression of APOE may have a more invasive capability because of increased cholesterol efflux, resulting in enhanced ERK and JNK signaling and AP-1-induced transcription of $M M P 7$ and other genes involved in tumor cell invasion.

$A P O E$ expression levels have also been found to be important in other cancer types. For example, Chen et $\mathrm{al}^{37}$ found that high-grade ovarian cancers demonstrated higher $A P O E$ expression compared to low-grade tumors. Their data suggested that $A P O E$ might be useful as a biomarker for other cancers, including breast, stomach, pancreatic, colon, and prostate. Sakashita et $\mathrm{al}^{38}$ found $A P O E$ to be a marker for invasion in gastric cancer, demonstrating that tumor samples with higher APOE mRNA expression exhibited more invasion into the muscular layer and a higher rate of lymph node metastases. And recently, Xu et $\mathrm{al}^{39}$ reported that serum levels of apolipoprotein E correlated with disease progression and poor prognosis in breast cancer patients.

Interestingly, higher APOE protein levels appear to have a suppressive effect on melanoma invasion and metastasis.
Pencheva et $\mathrm{al}^{40}$ characterized a model in melanoma whereby APOE protein acts through LDL receptor-related protein 1/ LDL receptor-related protein 8 receptors to suppress tumor invasion and metastasis. However, cholesterol levels were not examined in this study. In addition, APOE mRNA levels in melanoma cells are significantly higher than those observed in head and neck cancer cells. These differences suggest that the effect of APOE on cholesterol efflux and signaling via APOE receptors may be tumor type specific.

It is known that endogenous APOE contributes to cholesterol efflux to maintain a balance of cholesterol levels in the cell. $^{31,32,41}$ Consistent with this role, we found that knockdown of APOE led to an increase in total cholesterol, as well as a reduction in phosphorylation of ERK, JNK, and cJun. Addition of exogenous cholesterol to OSCC cells similarly resulted in dephosphorylation of ERK, supporting the hypothesis that increased cellular cholesterol can mediate the reduced phosphorylation of ERK, JNK, and cJun that we have observed in our study. There are several possible mechanisms by which increased cholesterol could inhibit ERK phosphorylation in OSCC cells. One possible mechanism of decreased ERK phosphorylation is through a cholesterol-mediated increase in the activity of the oxysterol-binding protein phosphatase complex. Oxysterol-binding protein is a protein previously implicated in lipid transport that is regulated by cholesterol and

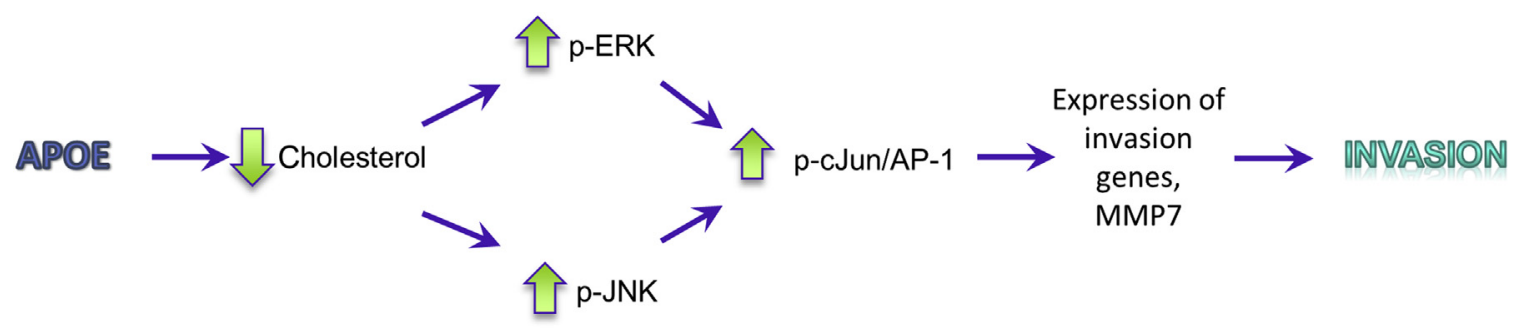

Figure 8 Proposed mechanism for the role of apolipoprotein E (APOE) in oral squamous cell carcinoma (OSCC) tumor invasion. High expression of APOE in OSCC tumor cells results in a decrease in total cellular cholesterol because of increased cholesterol efflux out of the cell. This decrease in cellular cholesterol results in increased phosphorylation of extracellular signal-regulated kinase (ERK) and JNK signaling proteins located downstream of membrane receptors. Increased phosphorylation of ERK and JNK leads to increased phosphorylation and activation of cJun, resulting in increased AP-1 transcription activity. AP-1 induces transcription of downstream target genes, such as MMP7, leading to an increase in tumor cell invasion. MMP, matrix metalloproteinase. 
controls ERK activation. $^{42-44}$ On binding cholesterol, oxysterol-binding protein binds both the serine/threonine phosphatase protein phosphatase $2 \mathrm{~A}$ and hematopoietic protein tyrosine phosphatase. This oligomer has dual Ser/Thr and Tyr phosphatase activity for ERK. A lowering of cellular cholesterol is accompanied by a disassembly of this oligomer, resulting in inactivation of the phosphatase and increased ERK activity.

An alternative mechanism for affecting tumor cell signaling could be the redistribution of growth factor receptors in the cell membrane in areas of high cholesterol content, termed lipid rafts. ${ }^{4-47}$ For example, it is known that the epidermal growth factor receptor can be localized in lipid rafts. ${ }^{4-50}$ Lipid rafts are also sites where receptor kinases for multiple signaling pathways converge, including the MAPK pathways. $^{33}$ Altering the cellular cholesterol concentration can result in a shift of membrane proteins, including receptors and membrane proteases, from raft to nonraft fractions, a transition that can decrease the activity of those proteins. ${ }^{50,51}$ Epidermal growth factor receptor is one such protein whose kinase activity is dependent on cholesterol. $^{36,52}$ Other signaling molecules that might be affected by this mechanism include transforming growth factor- $\beta^{35}$ and Lyn tyrosine kinase. ${ }^{53}$

The activity of the AP-1 transcription factor has been shown to be regulated by MAPKs, including ERK and JNK. ${ }^{54,55}$ IPA of transcriptome changes induced by $A P O E$ knockdown supported our findings with regard to ERK, Jnk, and Ap1 being the primary upstream regulators of the high $A P O E$ tumor phenotype. In addition, AP-1 activity has previously been correlated with tumor cell invasive capability. ${ }^{20,56}$ Intriguingly, we identified $M M P 7$ as one of the genes more highly expressed in the more invasive WPOI5 tumors, and MMP7 has been shown to be regulated by the AP-1 transcription factor. Similar results have been found for ovarian cancer invasion, ${ }^{22}$ metastatic potential of colon cancer cells, ${ }^{23}$ gastric cancer invasion, ${ }^{24}$ and breast cancer. ${ }^{25}$ These studies are consistent with a mechanism whereby APOE regulates MMP7 expression through AP-1.

Although our results reveal an intriguing mechanism linking APOE protein levels to OSCC tumor cell invasion, some limitations are seen in our study. First, although our initial screen for genes overexpressed in more aggressive (WPOI5) tumors revealed many novel candidates, these experiments were based on two microarray platforms and will need to be validated using an independent candidate approach, such as real-time PCR on independent OSCC cases. Second, the number of tumor samples examined for APOE immunohistochemical staining was small; clearly, a larger number of tumor tissue sections will need to be examined to make conclusions about the significance of APOE staining at the invading edge. Third, although the knockdown in tumor cell invasion with APOE knockdown was confirmed in a second OSCC cell line, we need to test whether the described mechanism is generalizable to other oral cavity cell lines, as well as other types of head and neck cancer. Future experiments will be needed to validate the effects of exogenous cholesterol on other parts of the signaling pathway, including cJun phosphorylation and AP1 activity. Further experiments will also be needed to validate the many downstream targets of APOE knockdown identified by the RNA-Seq experiments, test whether these changes are observed between WPOI5 and WPOI3 oral cavity tumors, and measure differences in cJun phosphorylation between these two tumor phenotypes.

In summary, we propose a model (Figure 8) where OSCC patients with invasive tumors have high levels of $A P O E$ expression that contribute to this invasive phenotype. High APOE protein levels increase cholesterol export and lower total cellular cholesterol, leading to an increase in MAPK signaling. Increased ERK and JNK signaling results in increased activity of AP-1, which, in turn, enhances the expression of genes involved in invasion, including the protease $M M P 7$. APOE expression may, therefore, serve as a useful prognostic marker in OSCC patients as a measure for tumor cells' propensity for invasion, and the targeting of its underlying mechanism could be exploited as a therapeutic target in the treatment of this disease.

\section{Acknowledgments}

We thank Dr. Ahmad Waseem (Queen Mary University of London, Barts, and the London School of Medicine and Dentistry) for kindly providing the AP-1 luciferase constructs; Dr. Thomas Carey (University of Michigan) for providing the UMSCC-1 cell lines; and the New York Genome Center for collaborating in RNA sequencing.

\section{Supplemental Data}

Supplemental material for this article can be found at http://dx.doi.org/10.1016/j.ajpath.2017.06.016.

\section{References}

1. Sasahira T, Kirita T, Kuniyasu H: Update of molecular pathobiology in oral cancer: a review. Int J Clin Oncol 2014, 19:431-436

2. Jimenez L, Jayakar SK, Ow TJ, Segall JE: Mechanisms of invasion in head and neck cancer. Arch Pathol Lab Med 2015, 139:1334-1348

3. Brandwein-Gensler M, Teixeira MS, Lewis CM, Lee B, Rolnitzky L, Hille JJ, Genden E, Urken ML, Wang BY: Oral squamous cell carcinoma: histologic risk assessment, but not margin status, is strongly predictive of local disease-free and overall survival. Am J Surg Pathol 2005, 29:167-178

4. Spiro RH, Huvos AG, Wong GY, Spiro JD, Gnecco CA, Strong EW Predictive value of tumor thickness in squamous carcinoma confined to the tongue and floor of the mouth. Am J Surg 1986, 152:345-350

5. Brandwein-Gensler M, Smith RV, Wang B, Penner C, Theilken A, Broughel D, Schiff B, Owen RP, Smith J, Sarta C, Hebert T, Nason R, Ramer M, DeLacure M, Hirsch D, Myssiorek D, Heller K, Prystowsky M, Schlecht NF, Negassa A: Validation of the histologic risk model in a new cohort of patients with head and neck squamous cell carcinoma. Am J Surg Pathol 2010, 34:676-688 
6. Li Y, Bai S, Carroll W, Dayan D, Dort JC, Heller K, Jour G, Lau H, Penner C, Prystowsky M, Rosenthal E, Schlecht NF, Smith RV, Urken M, Vered M, Wang B, Wenig B, Negassa A, Brandwein-Gensler M: Validation of the risk model: high-risk classification and tumor pattern of invasion predict outcome for patients with low-stage oral cavity squamous cell carcinoma. Head Neck Pathol 2013, 7:211-223

7. Almangush A, Bello IO, Coletta RD, Makitie AA, Makinen LK, Kauppila JH, Pukkila M, Hagstrom J, Laranne J, Soini Y, Kosma VM, Koivunen P, Kelner N, Kowalski LP, Grenman R, Leivo I, Laara E, Salo T: For early-stage oral tongue cancer, depth of invasion and worst pattern of invasion are the strongest pathological predictors for locoregional recurrence and mortality. Virchows Arch 2015, 467:39-46

8. Schlecht NF, Brandwein-Gensler M, Smith RV, Kawachi N, Broughel D, Lin J, Keller CE, Reynolds PA, Gunn-Moore FJ, Harris T, Childs G, Belbin TJ, Prystowsky MB: Cytoplasmic ezrin and moesin correlate with poor survival in head and neck squamous cell carcinoma. Head Neck Pathol 2012, 6:232-243

9. Lleras RA, Smith RV, Adrien LR, Schlecht NF, Burk RD, Harris TM, Childs G, Prystowsky MB, Belbin TJ: Unique DNA methylation loci distinguish anatomic site and HPV status in head and neck squamous cell carcinoma. Clin Cancer Res 2013, 19:5444-5455

10. Loudig O, Milova E, Brandwein-Gensler M, Massimi A, Belbin TJ, Childs G, Singer RH, Rohan T, Prystowsky MB: Molecular restoration of archived transcriptional profiles by complementary-template reverse-transcription (CT-RT). Nucleic Acids Res 2007, 35:e94

11. Loudig O, Brandwein-Gensler M, Kim RS, Lin J, Isayeva T, Liu C, Segall JE, Kenny PA, Prystowsky MB: Illumina whole-genome complementary DNA-mediated annealing, selection, extension and ligation platform: assessing its performance in formalin-fixed, paraffin-embedded samples and identifying invasion pattern-related genes in oral squamous cell carcinoma. Hum Pathol 2011, 42:1911-1922

12. Oser M, Yamaguchi H, Mader CC, Bravo-Cordero JJ, Arias M, Chen X, Desmarais V, van Rheenen J, Koleske AJ, Condeelis J: Cortactin regulates cofilin and N-WASp activities to control the stages of invadopodium assembly and maturation. J Cell Biol 2009, 186:571-587

13. Jimenez L, Sharma VP, Condeelis J, Harris T, Ow TJ, Prystowsky MB, Childs G, Segall JE: MicroRNA-375 suppresses extracellular matrix degradation and invadopodial activity in head and neck squamous cell carcinoma. Arch Pathol Lab Med 2015, 139:1349-1361

14. Brown L, Waseem A, Cruz IN, Szary J, Gunic E, Mannan T, Unadkat M, Yang M, Valderrama F, O'Toole EA, Wan H: Desmoglein 3 promotes cancer cell migration and invasion by regulating activator protein 1 and protein kinase C-dependent-Ezrin activation. Oncogene 2014, 33:2363-2374

15. Bentley DR, Balasubramanian S, Swerdlow HP, Smith GP, Milton J, Brown CG, et al: Accurate whole human genome sequencing using reversible terminator chemistry. Nature 2008, 456:53-59

16. Dobin A, Davis CA, Schlesinger F, Drenkow J, Zaleski C, Jha S, Batut P, Chaisson M, Gingeras TR: STAR: ultrafast universal RNAseq aligner. Bioinformatics 2013, 29:15-21

17. Liao Y, Smyth GK, Shi W: featureCounts: an efficient general purpose program for assigning sequence reads to genomic features. Bioinformatics 2014, 30:923-930

18. Love MI, Huber W, Anders S: Moderated estimation of fold change and dispersion for RNA-seq data with DESeq2. Genome Biol 2014, 15:550

19. Cancer Genome Atlas Network: Comprehensive genomic characterization of head and neck squamous cell carcinomas. Nature 2015, 517:576-582

20. Ozanne BW, Spence HJ, McGarry LC, Hennigan RF: Transcription factors control invasion: AP-1 the first among equals. Oncogene 2007, 26:1-10

21. Mburu YK, Egloff AM, Walker WH, Wang L, Seethala RR, van Waes C, Ferris RL: Chemokine receptor 7 (CCR7) gene expression is regulated by NF-kappaB and activator protein 1 (AP1) in metastatic squamous cell carcinoma of head and neck (SCCHN). J Biol Chem 2012, 287:3581-3590
22. Chang MC, Chen CA, Chen PJ, Chiang YC, Chen YL, Mao TL, Lin HW, Lin Chiang WH, Cheng WF: Mesothelin enhances invasion of ovarian cancer by inducing MMP-7 through MAPK/ERK and JNK pathways. Biochem J 2012, 442:293-302

23. Banskota S, Regmi SC, Kim JA: NOX1 to NOX2 switch deactivates AMPK and induces invasive phenotype in colon cancer cells through overexpression of MMP-7. Mol Cancer 2015, 14:123

24. Shi M, Liu D, Duan H, Han C, Wei B, Qian L, Chen C, Guo L, Hu M, Yu M, Song L, Shen B, Guo N: Catecholamine up-regulates MMP-7 expression by activating AP-1 and STAT3 in gastric cancer. Mol Cancer 2010, 9:269

25. Yuan G, Qian L, Song L, Shi M, Li D, Yu M, Hu M, Shen B, Guo N: Heregulin-beta promotes matrix metalloproteinase-7 expression via HER2-mediated AP-1 activation in MCF-7 cells. Mol Cell Biochem 2008, 318:73-79

26. Han JC, Li XD, Du J, Xu F, Wei YJ, Li HB, Zhang YJ: Elevated matrix metalloproteinase-7 expression promotes metastasis in human lung carcinoma. World J Surg Oncol 2015, 13:5

27. Mitsui H, Suarez-Farinas M, Gulati N, Shah KR, Cannizzaro MV, Coats I, Felsen D, Krueger JG, Carucci JA: Gene expression profiling of the leading edge of cutaneous squamous cell carcinoma: IL-24driven MMP-7. J Invest Dermatol 2014, 134:1418-1427

28. von Massenhausen A, Bragelmann J, Billig H, Thewes B, Queisser A, Vogel W, Kristiansen G, Schrock A, Bootz F, Brossart P, Kirfel J, Perner S: Implication of the receptor tyrosine kinase AXL in head and neck cancer progression. Int J Mol Sci 2016, 18:7

29. Katase N, Nishimatsu SI, Yamauchi A, Yamamura M, Terada K, Itadani M, Okada N, Hassan NMM, Nagatsuka H, Ikeda T, Nohno T, Fujita S: DKK3 overexpression increases malignant properties of head and neck squamous cell carcinoma cells. Oncol Res 2017, [Epub ahead of print] doi:10.3727/096504017X14926874596386

30. Bu J, Bu X, Liu B, Chen F, Chen P: Increased expression of tissue/salivary transgelin mRNA predicts poor prognosis in patients with oral squamous cell carcinoma (OSCC). Med Sci Monit 2015, 21: $2275-2281$

31. Langer C, Huang Y, Cullen P, Wiesenhutter B, Mahley RW, Assmann G, von Eckardstein A: Endogenous apolipoprotein E modulates cholesterol efflux and cholesteryl ester hydrolysis mediated by high-density lipoprotein-3 and lipid-free apolipoproteins in mouse peritoneal macrophages. J Mol Med (Berl) 2000, $78: 217-227$

32. Zhang WY, Gaynor PM, Kruth HS: Apolipoprotein E produced by human monocyte-derived macrophages mediates cholesterol efflux that occurs in the absence of added cholesterol acceptors. J Biol Chem 1996, 271:28641-28646

33. Furuchi T, Anderson RG: Cholesterol depletion of caveolae causes hyperactivation of extracellular signal-related kinase (ERK). J Biol Chem 1998, 273:21099-21104

34. Kim S, Han J, Lee DH, Cho KH, Kim KH, Chung JH: Cholesterol, a major component of caveolae, down-regulates matrix metalloproteinase-1 expression through ERK/JNK pathway in cultured human dermal fibroblasts. Ann Dermatol 2010, 22: 379-388

35. Chen CL, Liu IH, Fliesler SJ, Han X, Huang SS, Huang JS: Cholesterol suppresses cellular TGF-beta responsiveness: implications in atherogenesis. J Cell Sci 2007, 120:3509-3521

36. Ringerike T, Blystad FD, Levy FO, Madshus IH, Stang E: Cholesterol is important in control of EGF receptor kinase activity but EGF receptors are not concentrated in caveolae. J Cell Sci 2002, 115:1331-1340

37. Chen YC, Pohl G, Wang TL, Morin PJ, Risberg B, Kristensen GB, Yu A, Davidson B, Shih Ie M: Apolipoprotein E is required for cell proliferation and survival in ovarian cancer. Cancer Res 2005, 65:331-337

38. Sakashita K, Tanaka F, Zhang X, Mimori K, Kamohara Y, Inoue H, Sawada T, Hirakawa K, Mori M: Clinical significance of ApoE expression in human gastric cancer. Oncol Rep 2008, 20:1313-1319

39. Xu X, Wan J, Yuan L, Ba J, Feng P, Long W, Huang H, Liu P, Cai Y, Liu M, Luo J, Li L: Serum levels of apolipoprotein E correlates with 
disease progression and poor prognosis in breast cancer. Tumour Biol 2016, 37:15959-15966

40. Pencheva N, Tran H, Buss C, Huh D, Drobnjak M, Busam K, Tavazoie SF: Convergent multi-miRNA targeting of ApoE drives LRP1/LRP8-dependent melanoma metastasis and angiogenesis. Cell 2012, 151:1068-1082

41. Ikonen E: Cellular cholesterol trafficking and compartmentalization. Nat Rev Mol Cell Biol 2008, 9:125-138

42. Olkkonen VM, Li S: Oxysterol-binding proteins: sterol and phosphoinositide sensors coordinating transport, signaling and metabolism. Prog Lipid Res 2013, 52:529-538

43. Wang PY, Weng J, Anderson RG: OSBP is a cholesterol-regulated scaffolding protein in control of ERK 1/2 activation. Science 2005, 307:1472-1476

44. Wang PY, Weng J, Lee S, Anderson RG: The N terminus controls sterol binding while the $\mathrm{C}$ terminus regulates the scaffolding function of OSBP. J Biol Chem 2008, 283:8034-8045

45. Orr G, Hu D, Ozcelik S, Opresko LK, Wiley HS, Colson SD: Cholesterol dictates the freedom of EGF receptors and HER2 in the plane of the membrane. Biophys J 2005, 89:1362-1373

46. de Laurentiis A, Donovan L, Arcaro A: Lipid rafts and caveolae in signaling by growth factor receptors. Open Biochem J 2007, 1:12-32

47. Freeman MR, Cinar B, Kim J, Mukhopadhyay NK, Di Vizio D, Adam RM, Solomon KR: Transit of hormonal and EGF receptordependent signals through cholesterol-rich membranes. Steroids 2007, 72:210-217

48. Balbis A, Posner BI: Compartmentalization of EGFR in cellular membranes: role of membrane rafts. J Cell Biochem 2010, 109:1103-1108
49. Pike LJ, Han X, Gross RW: Epidermal growth factor receptors are localized to lipid rafts that contain a balance of inner and outer leaflet lipids: a shotgun lipidomics study. J Biol Chem 2005, 280: 26796-26804

50. Saffarian S, Li Y, Elson EL, Pike LJ: Oligomerization of the EGF receptor investigated by live cell fluorescence intensity distribution analysis. Biophys J 2007, 93:1021-1031

51. Schmidt V, Carlo AS, Willnow TE: Apolipoprotein E receptor pathways in Alzheimer disease. Wiley Interdiscip Rev Syst Biol Med 2014, 6:255-270

52. Westover EJ, Covey DF, Brockman HL, Brown RE, Pike LJ: Cholesterol depletion results in site-specific increases in epidermal growth factor receptor phosphorylation due to membrane level effects: studies with cholesterol enantiomers. J Biol Chem 2003, 278: $51125-51133$

53. Morinaga T, Abe K, Nakayama Y, Yamaguchi N, Yamaguchi N: Activation of Lyn tyrosine kinase through decreased membrane cholesterol levels during a change in its membrane distribution upon cell detachment. J Biol Chem 2014, 289:26327-26343

54. Eferl R, Wagner EF: AP-1: a double-edged sword in tumorigenesis. Nat Rev Cancer 2003, 3:859-868

55. Chakraborty A, Diefenbacher ME, Mylona A, Kassel O, Behrens A: The E3 ubiquitin ligase Trim7 mediates c-Jun/AP-1 activation by Ras signalling. Nat Commun 2015, 6:6782

56. Kajanne R, Miettinen P, Mehlem A, Leivonen SK, Birrer M, Foschi M, Kahari VM, Leppa S: EGF-R regulates MMP function in fibroblasts through MAPK and AP-1 pathways. J Cell Physiol 2007, 212:489-497 